\author{
A. E. Malcolm 1,* \\ Department of Earth Sciences, Utrecht University \\ F. Reitich and J. Yang \\ Department of Mathematics, University of Minnesota
}

J. F. Greenleaf and M. Fatemi

Mayo Clinic College of Medicine

\title{
A combined parabolic-integral equation approach to the acoustic simulation of vibro-acoustic imaging
}

\begin{abstract}
This paper aims to model ultrasound vibro-acoustography to improve our understanding of the underlying physics of the technique thus facilitating the collection of better images. Ultrasound vibro-acoustography is a novel imaging technique combining the resolution of high-frequency imaging with the clean (speckle-free) images obtained with lower frequency techniques. The challenge in modeling such an experiment is in the variety of scales important to the final image. In contrast to other approaches for modeling such problems, we break the experiment into three parts: high frequency propagation, nonlinear interaction and the propagation of the lowfrequency acoustic emission. We then apply different modeling strategies to each
\end{abstract}


part. For the high-frequency propagation we choose a parabolic approximation as the field has a strong preferred direction and small propagation angles. The nonlinear interaction is calculated directly with Fourier methods for computing derivatives. Because of the low-frequency omnidirectional nature of the acoustic emission field and the piecewise constant medium we model the low-frequency field with a surface integral approach. We use our model to compare with experimental data and to visualize the relevant fields at points in the experiment where laboratory data is difficult to collect, in particular the source of the low-frequency field. To simulate experimental conditions we perform the simulations with the two frequencies 3 and $3.05 \mathrm{MHz}$ with an inclusion of varying velocity submerged in water.

Key words: ultrasound vibro-acoustography, one-way wave equation, integral methods, radiation force, numerical modeling

PACS: 02.30.Rz, 02.60.Cb, 43.25.Qp

\section{Introduction}

Ultrasound vibro-acoustography was developed $[1,2]$ to achieve the high-resolution possible with high-frequency imaging modalities without the artifacts, caused by scattering from small inclusions, present in high-frequency images. The experiment, which is discussed in more detail in [1,2], breaks naturally into three parts as illustrated in Figure 1. The first is the focusing of two beams of ultrasound at two slightly different high (a few $\mathrm{MHz}$ ) frequencies, $f_{1}$ and $f_{2}$ (or $\omega_{1}$ and $\omega_{2}$ for angular frequency), on the region of interest. These two

* Corresponding author Email address: amalcolm@mit.edu (A. E. Malcolm ).

1 Now at: Department of Earth, Atmospheric and Planetary Sciences, Massachusetts Institute of Technology 
fields then interact nonlinearly to create a force at the difference frequency $\Delta \omega=\omega_{1}-\omega_{2}$ (a few tens of $\mathrm{kHz}$ ). The third step is the propagation of the excited low-frequency field to a hydrophone located far from the focus point. Similar experiments have been modeled with the finite element method [3,4], without separating these scales; here we separate the different scales, through a perturbation approach, for increased computational efficiency. This approach was first discussed in [5] in which preliminary results were shown where all of the modeling was done with a parabolic approximation. In [6] we further develop this methodology introducing integral methods for modeling the lowfrequency emission and exploring the validity of 2D methods for modeling this problem. In the present paper we summarize our modeling approach and focus on the benefits of modeling in understanding the imaging modality.

Following the description of the experiment given in $[1,2]$, the two highfrequency fields are described by a first-order deviation from a background state and propagate a few hundred wavelengths before interacting with an object only a few wavelengths in size. The first property is difficult to accommodate in full-wave methods and the second in high-frequency approximations. The fields have a strong preferred direction (away from the transducer), however, and the velocity variations in tissue are relatively small, making oneway or parabolic approximations to the wave equation a good option for these high-frequency fields. In such approximations, a preferred axis of propagation is defined and waves are propagated in only one direction with respect to this axis. The resulting wavefield is more accurate than high-frequency approximations such as ray theory, but faster to compute than full-wave methods such as finite-difference, finite element or integral methods. An introduction to these methods is found in $[7,8]$; these approximations have been used to simulate 
waves in tissue in [9].

It is the nonlinear interaction of these two high-frequency fields that is the source of the low-frequency waves recorded to form an image. This interaction is described by a second-order correction to the wave equation for a secondorder deviation from the background state derived, for example, in $[10,11]$ and related to the so-called radiation force or parametric radiation force. The idea of mixing two high-frequency beams to generate a low-frequency signal was suggested by [12] in extending his own work (see e.g. [13-15]) on the radiation force. The technique discussed here is a refinement of this early suggestion for the exploitation of the radiation force to generate low-frequency signal. The ideas rest upon the same physics but in ultrasound vibro-acoustography, the low-frequency field is generated only in a small region from the interaction of two focused beams. We choose to use the differential model rather than the integral force model because it is straightforward to implement and it describes the relevant physics of the imaging technique. By recording only at the difference frequency, the experiment ensures that only waves excited by this nonlinear interaction are recorded. A key point in the design of ultrasound vibro-acoustography is that the size of the focal area and thus the resolution are governed by the high frequency.

The source of the low-frequency field is point-like, resulting in a relatively omni-direction field at $\Delta \omega$, precluding the use of the parabolic approximation in its computation. We choose an integral method to model this part of the experiment because of the low-frequency of the field as compared with the size of the inclusion $(50 \mathrm{kHz}$ field and an inclusion approximately $3 \mathrm{~cm}$ in radius) and because an image is formed of several inclusions, each with constant acoustic velocity, embedded in a background medium also with constant 


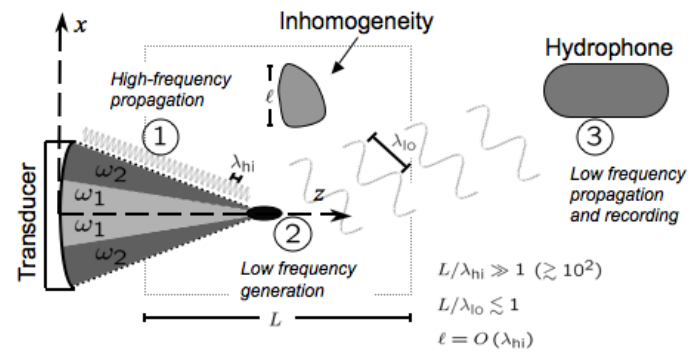

Fig. 1. Schematic of the experimental setup, as described in $[1,2]$.

velocity. More specifically, we choose a surface integral method as discussed in [16] which, along with [17] gives a good introduction to integral methods.

\section{Experiment and Model}

In the first step of the experiment, the high-frequency field is excited by a confocal spherical transducer positioned so that the focal point is within the region of interest; the inner circle of this transducer excites waves at one frequency, $f_{1}$ and the outer annulus at another frequency, $f_{2}$. Following [18], we model the transducer as a set of point sources so that, for example, in two dimensions the simulated transducer consists of point sources arranged on a circle. Although they rely on similar physics, as mentioned in the introduction, the focused ultrasound beam used in $[1,2]$ and modeled here differs from the parametric arrays introduced by Westervelt [12] in that the low-frequency field is generated in the vicinity of a small focal region within the region of interest rather than over an extended region.

The two frequencies excited by the transducer, satisfy the acoustic wave equation

$$
\partial_{t}^{2} \phi_{j}-c(x)^{2} \triangle \phi_{j}=0, \quad j=1,2,
$$

where $c(x)$ is the (isotropic) acoustic velocity and $\phi$ is the first-order velocity 
potential (related to the particle velocity by $v=\nabla \phi$ ) considered as a perturbation from a background state. To explain the generation of the acoustic emission at the difference frequency, $\Delta \omega$, we extend the linear wave-equation to

$$
\partial_{t}^{2} \psi-c^{2} \triangle \psi=\partial_{t}\left[|\nabla \phi|^{2}+\frac{\gamma-1}{2 c^{2}}\left|\partial_{t} \phi\right|^{2}\right],
$$

with $\phi=\phi_{1}+\phi_{2}, \psi$ the velocity potential associated with a second-order perturbation from the background state, and $\gamma$ describing the nonlinearity of the medium. Equation (2) is derived (see e.g. [10,11]) from the Euler equations of motion, and the equation of continuity, under the constant entropy assumption, which gives the constitutive relation

$$
p=p_{0}\left(\frac{\rho}{\rho_{0}}\right)^{\gamma}
$$

The pressure, at the difference frequency, associated with the velocity potential, $\psi$, is recorded with a hydrophone; an image is then formed by plotting the amplitude of $\psi$ as a function of the position of the focal point. The squared terms on the right-hand side of equation (2) result in the excitation of waves at several different frequencies. In the experiment, the field resulting from this excitation is recorded using a hydrophone sensitive to the difference frequency, $\Delta \omega$, making only this component important in the calculations. As this frequency is present only in $\psi$ and not in $\phi_{1,2}$, the difference in pressure from the unperturbed state is well approximated by $\psi$. The right-hand side of equation (2) is related to the parametric radiation force in which the first term constitutes the classical radiation force. By simulating the differential equation explicitly, however, we do not enforce that the characteristics of the image are governed entirely by the structure of this forcing but also include explicitly the possibility that the variations in the image amplitude are a direct result of the scattering of the low-frequency field. To relate the right-hand side 
of equation 2 to the radiation force, we note that from the discussion in both [19] and [11], we expect the sum of these two responses to be the time average of the integral, over the surface of the inclusion, of the difference between the pressure in the perturbed and unperturbed states, here $\psi$.

Equations (1) and (2) give a complete, acoustic, model of the experiment. An acoustic model is not expected to be sufficient for tissue modeling and an elastic or even visco-elastic model would improve the model. The extension of equation (2) to the visco-elastic case is discussed in [20]; extending our full methodology to this case is left for future work.

\section{Numerical Algorithm}

The challenge of developing algorithms for UVA is in the exploitation of the specific properties of the wave propagation over the range of scales present. A first simplification comes in recognizing that there are three frequencies involved in the experiment, $\omega_{1,2}$ and $\Delta \omega$; to exploit this sparsity we perform all of the calculations in the temporal frequency domain. Each step of the experiment requires a separate modeling strategy as discussed in the following subsections.

\subsection{High-frequency modeling}

As mentioned in the introduction, one-way methods are ideal for the highfrequency fields as they allow the accurate propagation over many wavelengths with better accuracy than ray methods for scattering from small obstacles. The idea behind one-way methods is to choose a preferred direction, $z$, of 
propagation (here away from the transducer along its axis) and then re-write the wave-equation as a first-order system of partial differential equations in this variable. This system is then diagonalized, analogous to the diagonalization of a matrix, to split the wavefield $\phi$, into two parts, $\phi_{ \pm}$, with $\phi_{+}$containing waves that propagate in the positive $z$ direction and $\phi_{-}$waves propagating in the negative $z$ direction. One-way methods then solve only one of the resulting two equations,

$$
\partial_{z} \phi_{+}+i A \phi_{+}=0
$$

where $A^{2}=-\partial_{x}^{2}-\frac{\omega^{2}}{c(x, z)^{2}}$. If $c(x, z)$ is independent of $x$, this equation can be solved exactly by taking the Fourier transform (in $x$ ) of the wavefield at depth $z$, multiplying by a phase-shift term

$$
e^{i\left(z-z^{\prime}\right) \omega \sqrt{c_{0}^{-2}(z)-\left\|k_{x}\right\|^{2}}}
$$

where $k_{x}$ is the lateral wavenumber and then taking the inverse Fourier Transform obtaining the field at depth $z^{\prime}$. This process is repeated until the wavefield has been calculated at all $z$ of interest. In this experiment, we assume that the background velocity is piecewise constant, with a single velocity for the background medium and another velocity for each obstacle. In this case, we can use a simplification of the phase-shift-plus-interpolation method of [21] in which the field is propagated from $z$ to $z^{\prime}$ at each of the velocities and the final field is obtained in the space domain (after inverse Fourier transform) by taking the field propagated with the correct velocity for that spatial point.

There are two limitations to this type of approximation, which are discussed in e.g. [7]. The first is the maximum angle, $\theta$ (measured away from the transducer axis) to which the field can be accurately computed and the second is the maximum lateral change in velocity that can be accurately dealt with. In 
the first case the accuracy is readily calculated, in a constant velocity model, as the error is in ignoring the so-called $Q$ operators discussed in [22] which

amounts to division by $\sqrt{\cos (\theta)}$. For variable velocities we find approximately a $1 \%$ error with a $10 \%$ velocity contrast; this error grows rapidly as the backscattered field becomes stronger as can be seen by examining the reflection and transmission coefficients and noting that the reflected field is ignored.

\subsection{Nonlinear Interaction}

The nonlinear interaction given on the right-hand side of equation (2) is relatively easy to compute directly. As the entire calculation is done in the temporal frequency domain, the time derivatives are computed through multiplication with $i \omega$. For the spatial derivatives, we first taper the high-frequency fields to zero at the boundaries of the computational domain and then compute the derivatives in the spatial frequency domain.

An alternative approach to the simulation of both the high-frequency propagation and the nonlinear interaction is the TAWE method suggested in [23]. This method, which builds on a model suggested in [24], uses a similar strategy to the so-called split-step techniques in one-way methods. Instead of a correction for a variable velocity, however, these works include a correction for the nonlinearity of the medium. They calculate, on the fly, a simplified version of the right-hand side of equation (2) in which the gradient term is ignored as justified in [25]. As it is fully nonlinear, the method solves directly for, in our notation, both $\phi$ and $\psi$. This full nonlinearity, however, has rather dramatic effects on the associated computational cost (as it leads to an operation count that grows quintically with the number of frequencies considered in 
the calculation [24]) which can be shown to be significantly higher than that of the methodology proposed herein, although the difference in complexity with respect to the number of spatial degrees of freedom is less clear. It is this relative efficiency, along with the ability to look at intermediate results, such as the high-frequency fields and their nonlinear interactions, that is behind our choice of the three-step perturbative approach. In addition, the TAWE method is limited to layered media and, in our case, the gradient term may be significant and the velocity model will certainly deviate from the layered case. Finally, because we model a continuous wave experiment the issues associated with computing a tone-burst as discussed in [23] are not relevant at this time, although they may be a factor in future studies (and may increase the computational complexity of our method).

\subsection{Low-frequency modeling}

Integral equation methods rely on knowledge of the Green's function both within and outside the inclusions, and on using this Green's function to rewrite the differential equations describing the system as integral equations. Denoting by $G_{i}$ the Green's function inside the inclusions and by $G_{e}$ the Green's function outside the inclusions, we arrive at the system of equations

$$
\begin{gathered}
\psi_{e}(x)=\psi^{i n c}(x)+\int_{\partial D} \frac{\partial\left(G_{i}-G_{e}\right)}{\partial n_{y}}(x, y) \psi_{e}(y) \\
-\left(G_{i}-G_{e}\right)(x, y) \frac{\partial \psi_{e}}{\partial n_{y}}(y) d y \quad x \in \partial D, \\
\frac{\partial \psi_{e}}{\partial n_{x}}(x)=\frac{\partial \psi^{i n c}}{\partial n_{x}}(x)+\int_{\partial D} \frac{\partial^{2}\left(G_{i}-G_{e}\right)}{\partial n_{x} \partial n_{y}}(x, y) \psi_{e}(y) \\
-\frac{\partial_{x}\left(G_{i}-G_{e}\right)}{\partial n_{x}}(x, y) \frac{\partial \psi_{e}}{\partial n_{y}}(y) d y \quad x \in \partial D,
\end{gathered}
$$


for the second-order velocity potential, $\psi$, and its normal derivative where the normal is directed outward from the inclusions into the surroundings. In the above equations a superscript inc denotes the incident field (field without inclusions). We define $\psi^{i n c}=\psi_{i}^{i n c}+\psi_{e}^{i n c}$ where

$$
\begin{aligned}
& \psi_{i}^{i n c}(x)=\int_{D} G_{i}(x, y) f(y) d y \quad x \in \mathbb{R}^{2}, \\
& \psi_{e}^{i n c}(x)=\int_{D^{c}} G_{e}(x, y) f(y) d y \quad x \in \mathbb{R}^{2},
\end{aligned}
$$

with $f(y)$ the right-hand side of equation (2). Note that equations (6) and (7) are defined on the boundary of the inclusions rather than on the inclusions themselves, reducing the dimensionality of the problem and thus allowing faster computations. The solution of the equations $(6),(7),(8)$ is complicated by the singularities in the Green's functions; to account for this, we use the spectral quadratures defined by Colton and Kress [16, pp. 67-78] to discretize these integrals. These methods can be further accelarated to improve computational performance (e.g. via FFTs [26,27], Fast Multipole Methods [28], etc).

\section{Results}

In this section we demonstrate the utility of the model developed above. We use the experimental configuration depicted in Figure 2; the inhomogeneity is characterized by an acoustic velocity different from the surrounding (water) medium and has a diameter of $3 \mathrm{~mm}$. All of the axis labels are given in terms of the wavelength $\left(\lambda_{\mathrm{HI}}=0.5 \mathrm{~mm}\right.$ ), in water (with velocity $1.5 \mathrm{~mm} / \mu \mathrm{s}$ ), at $3 \mathrm{MHz}$; the two frequencies are 3 and $3.05 \mathrm{MHz}$. In the notation used in the previous section, the "image" is formed by plotting $|\psi|$ as a function of the 


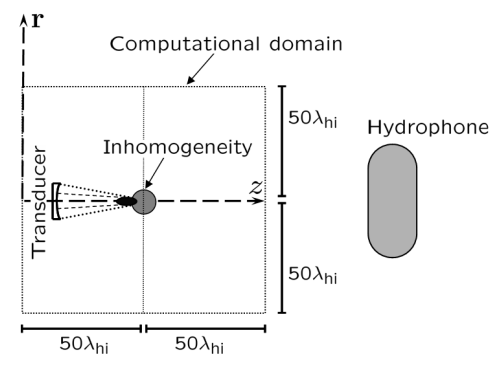

Fig. 2. Experimental configuration for both computational experiments shown below.

focal point.

In our first experiment, we explore the changes in the image when the inner and outer frequencies are interchanged; see Figure 3. We use a relatively small velocity contrast of $1.65 \mathrm{~mm} / \mu$ s to ensure the validity of the parabolic approximation and to mimic the relatively small contrasts found in different types of tissue and a relatively large transducer aperture of $120^{\circ}$ to make the clearest image possible. Note that while the absolute value of the secondary, low-frequency source remains relatively unchanged the phase, and thus the image, changes drastically.

In our second experiment, which is compared to laboratory data, a cylindrical Aluminum rod long in the $y$-direction was submerged in water and an image was made of this rod using a transducer with an aperture of $30^{\circ}$; a comparison of real and simulated images is shown in Figure 4. Aluminum has a relatively fast velocity of $6 \mathrm{~mm} / \mu \mathrm{s}$, beyond the expected range in medical imaging applications and also beyond the range where the parabolic approximation can be guaranteed to be accurate. Despite these limitations we have captured the basic structure of the image. 

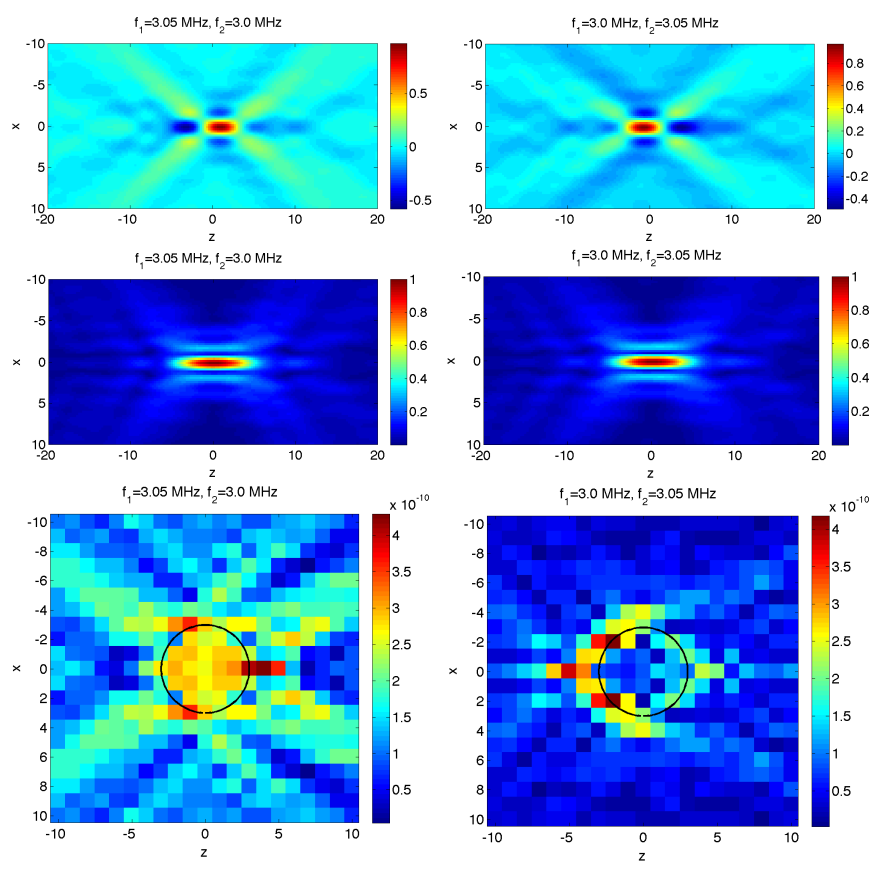

Fig. 3. The upper plots show the real part of the right-hand side of equation (refeq:RHS), the middle plots the absolute value, and the lower plots images. The frequencies $f_{1}$ and $f_{2}$ are interchanged between the left and right columns.

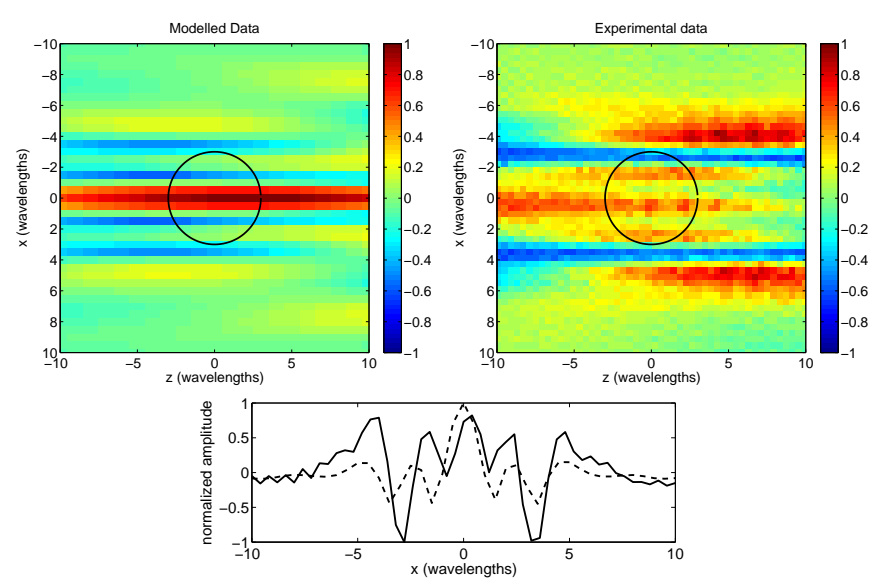

Fig. 4. Upper left is the synthesized image, on the upper right is the experimental image. Below is a comparison of a slice of the image for fixed $z=0$.

\section{Discussion and Conclusions}

We have presented an acoustic model for ultrasound vibro-acoustography that first uses a perturbation argument to break the experiment into three steps 
and then employs both exact methods and appropriate approximations, to model the relevant physics of each step, for computational efficiency. With this tool we are able to visualize the relevant wavefields at several stages in the imaging process. Of particular importance is the ability to examine the characteristics of the (high-frequency generated) source of the low frequency excitation, the measurement of which is not straightforward in the laboratory. We have demonstrated the ability of our model to capture the broad features of the experimental data despite the limitation of our current model to $2 \mathrm{D}$ acoustic materials. Future research along the lines presented here includes the extension of this model to 3D and to visco-elastic materials to more accurately simulate experimental configurations of clinical interest.

\section{ACKNOWLEDGMENTS}

We are grateful for many useful discussions in particular with respect to the radiation force with Farid Mitri of the Mayo clinic. We also thank Randall Kinnick for collecting the experimental data. This work was supported in part by Grants CA 127235 and CA 91956 from the National Institute of Health, and by the Dutch National Science Foundation grant number NWO:VIVI865.03.007.

\section{References}

[1] M. Fatemi, J. F. Greenleaf, Ultrasound stimulated vibro-acoustic spectrography, Science 280 (1998) 82-85.

[2] M. Fatemi, J. F. Greenleaf, Vibro-acoustography: An imaging modality based on ultrasound-stimulated acoustic emission, Proc. Natl. Acad. Sci. USA 96 
(1999) 6603-6608.

[3] J. Heikkilä, T. Karjalainen, M. Vaukonen, K. Hynynen, 3D simulations of difference frequency effects on a blood vessel in ultrasound-stimulation vibroacoustography, 2004, IEEE Ultrasonics Symposium (2004) 1247-1250.

[4] J. C. Brigham, W. Aquino, F. G. Mitri, J. F. Greenleaf, M. Fatemi, Inverse estimation of viscoelastic material properties for solids immersed in fluids using vibroacoustic techniques, Journal of Applied Physics 101 (2) (2007) 023509.

[5] A. E. Malcolm, F. Reitich, J. Yang, M. Fatemi, J. F. Greenleaf, A complete computational model of ultrasound vibro-acoustography, No. IMECE200616385, ASME International Mechanical Engineering Congress and Exposition, 2006.

[6] A. E. Malcolm, F. Reitich, J. Yang, J. F. Greenleaf, M. Fatemi, Numerical modeling for assessment and design of ultrasound vibroacoustography systems, in: M. Fatemi, A. Al-Jumaily (Eds.), Biomedical Applications of Vibration and Acoustics for Imaging and Characterizations, ASME Press, 2008.

[7] F. D. Tappert, The parabolic approximation method, in: J. B. Keller, J. S. Papadakis (Eds.), Wave Propagation and Underwater Acoustics, SpringerVerlag, Berlin, 1977.

[8] F. Jensen, W. Kuperman, M. Porter, H. Schmidt, Computational Ocean Acoustics, Series in Modern Acoustics and Signal Processing, AIP, 2000.

[9] T. Varslot, S.-E. Måsøy, Forward propagation of acoustic pressure pulses in 3D soft biological tissue, Modeling, Identification and Control 27 (3) (2006) 181.

[10] H. Olsen, W. Romberg, H. Wergeland, Radiation force on bodies in a sound field, J. Acoust. Soc. Am. 30 (1) (1958) 69-76.

[11] T. Hasegawa, T. Kido, T. Iizuka, C. Matsuoka, A general theory of Rayleigh and Langevin radiation pressures, J. Acoust. Soc. Jpn. (E) 21 (3) (2000) 145-152. 
[12] P. J. Westervelt, Parametric acoustic array, The Journal of the Acoustical Society of America 35 (4) (1963) 535-537.

URL http://link.aip.org/link/?JAS/35/535/1

[13] P. J. Westervelt, The theory of steady forces caused by sound waves, The Journal of the Acoustical Society of America 23 (3) (1951) 312-315.

URL http://link.aip.org/link/?JAS/23/312/1

[14] P. J. Westervelt, Scattering of sound by sound, The Journal of the Acoustical Society of America 29 (2) (1957) 199-203.

URL http://link.aip.org/link/?JAS/29/199/1

[15] P. J. Westervelt, Scattering of sound by sound, The Journal of the Acoustical Society of America 29 (8) (1957) 934-935.

URL http://link.aip.org/link/?JAS/29/934/1

[16] D. Colton, R. Kress, Inverse Acoustic and Electromagnetic Scattering, second edition, Springer, 1998.

[17] D. Colton, R. Kress, Integral Equation Methods in Scattering Theory, Springer, 1983.

[18] B. Piwakowski, K. Sbai, A new approach to calculate the field radiated from arbitrarily structured transducer arrays, IEEE Transactions on Ultrasonics, Ferroelectrics and Frequency Control 46 (2) (1999) 422-440.

[19] G. R. Torr, The acoustic radiation force, Am. J. Phys. 52 (1984) 402-408.

[20] S. Callé, J.-P. Remenieéras, F. Pata, O. Matar, Application of vibroacoustography to tissue elasticity imaging, Acta Acustica United with Acustica 89 (2003) 936-941.

[21] J. Gazdag, P. Sguazzero, Migration of seismic data by phase shift plus interpolation, Geophysics 49 (2) (1984) 124-131. 
[22] C. C. Stolk, A pseudodifferential equation with damping for one-way wave propagation in inhomogeneous acoustic media, Wave Motion 40 (2004) 111121.

[23] J. Wójcik, A. Nowicki, P. Lewin, P. Bloomfield, T. Kujawska, L. Filipczynski, Wave envelopes method for description of nonlinear acoustic wave propagation, Ultrasonics 44 (3) (2006) 310-329.

[24] P. T. Christopher, K. J. Parker, New approaches to nonlinear diffractive field propagation, The Journal of the Acoustical Society of America 90 (1) (1991) 488-499.

URL http://link.aip.org/link/?JAS/90/488/1

[25] J. Wójcik, Conservation of energy and absorption in acoustic fields for linear and nonlinear propagation, The Journal of the Acoustical Society of America 104 (5) (1998) 2654-2663.

URL http://link.aip.org/link/?JAS/104/2654/1

[26] E. Bleszynski, M. Bleszynski, T. Jaroszewicz, AIM: Adaptive integral method for solving large-scale electromagnetic scattering and radiation problems, Radio Sci. 31 (1996) 1225.

[27] X. M. Xu, Q. H. Liu, Fast spectral-domain method for acoustic scattering problems, IEEE T. Ultrason. Ferr. 48 (2001) 522-529.

[28] R. Coifman, V. Rokhlin, S. Wandzura, The fast multiple method for the wave equation: a pedestrian prescription, IEEE Ant. Propagat. Mag. 35 (1993) 7-12. 